\title{
On the Riemann-Hilbert Problem with a Piecewise Constant Matrix
}

\author{
Vladimir V. Mityushev and Sergei V. Rogosin
}

\begin{abstract}
A constructive solution to the Riemann-Hilbert boundary value problem as well as to the $\mathbb{C}$-linear conjugation problem (or Riemann problem) with a special piecewise constant matrix for a multiply connected domain is obtained. The result is based on a vector generalization of the scalar method of functional equations in complex domain and on application of the Christoffel-Schwarz transformation of the half-plane onto a circular polygon.
\end{abstract}

Keywords. Riemann-Hilbert boundary value problem, vector-matrix $\mathbb{C}$-linear conjugation problem, multiply connected domain, Christoffel-Schwarz transformation, functional equation

Mathematics Subject Classification (2000). 30E25, 45G05

\section{Introduction}

The aim of this paper is the construction of solution to the vector-matrix Riemann boundary value problem for circular multiply connected domains. We develop here the proposed method only for the case of constant matrix coefficients on each connected component of the boundary under some restrictions, but it can be done also for more general coefficients. The main idea of the method is the reduction to a system of functional equations in complex domain as generalization of the results for scalar case presented in the monograph [25]. We restrict our considerations to the model case in order to get the solution in the most simple form which is very important for applications.

The solvability of boundary value problems with matrix coefficients has connection to different problems of analysis. The most natural of them are Fredholm (Noether) properties of the singular integral operators [9, 10, 17], and the solvability of the systems of singular integral equations [16, 29]. One of the

V. V. Mityushev: Department of Mathematics, Krakow Pedagogical Academy, ul. Podchorazych 2, 30-084 Krakow, Poland; vmityu@yahoo.com

S. V. Rogosin: Faculty of Mathematics and Mechanics, Belarusian State University, Nezavisimosti ave, 4, 220030 Minsk, Belarus; rogosin@bsu.by 
basic method for studying these problems is a factorization of matrix-functions in different function spaces settings as developed in [23] (see also the recent contribution by Ehrhardt and Spitkovsky [11] and references therein).

The factorization of matrix functions is closely related to the question on existence and construction of a linear differential equation with given singular points and a given monodromy group (for extended exposition on this relationship see, e.g., [11]). In Fuchsian case (i.e., when all given singularities are simple poles) the latter problem coincides with celebrated 21st Hilbert problem (or the Riemann-Hilbert problem) (see, e.g., [5]). Existence seemed to be proven by Plemelj in 1908 [27]. His idea was the reduction of the factorization problem to the following homogeneous boundary value problem

$$
\Phi^{+}(t)=G(t) \Phi^{-}(t), \quad t \in \Gamma,
$$

with respect to vectors $\Phi^{+}(z), \Phi^{-}(z)$ analytic in domains $D^{+}=\operatorname{int} \Gamma, D^{-}=$ $\operatorname{ext} \Gamma$, respectively. Here, $\Gamma$ is a piecewise linear contour connecting the given singular points and $G(t)$ is a piecewise constant matrix, which is constructed on the basis of the given monodromy. The problem (1.1) is usually called vectormatrix Riemann boundary value problem (or $\mathbb{C}$-linear conjugation problem with a matrix coefficient). Much later it was shown that the proof by Plemelj is not complete in the general case (but it is still correct in the case when at least one of the monodromy matrices can be diagonalized, see, e.g., [3]). Description of the negative solution to the 21st Hilbert problem is presented in [5]. In contrast to the Fuchsian linear systems, a similar problem for the class of systems with regular singular points has always a positive solution (cf., e.g., [6]). The $\mathbb{C}-$ linear conjugation problem (Riemann problem) (1.1) is closely related to the following one (Riemann-Hilbert problem):

$$
\operatorname{Re}\{G(t) \Phi(t)\}=0, \quad t \in \Gamma,
$$

with respect to $\Phi(z)$ analytic in $D^{+}$.

The problem we consider here is a special case of the problem (1.1). Let $E$ be a unit matrix. The essential restriction is that in our study the matrix $G(t)$ in (1.1) satisfies the condition

$$
G^{2}(t)=E, \quad t \in \Gamma
$$

and $G(t)=E$ on certain components of $\Gamma$ (hence we deal with the problem for multiply connected domain). The problem (1.2) is solved without the restriction (1.3). Therefore, we are always in the situation when the existence proof by Plemelj is still valid. We only present a constructive solution to boundary value problems, and thus a construction of a factorization problem in the above said special case. 
Among the applications of the theory of vector-matrix boundary value problems (as well as Riemann-Hilbert problem and factorization of matrix functions) we have to point out those in the elasticity theory $[19,20,32]$, and in the related to it diffraction problems [24], in the quantum gravity [13], and in the approximation theory [2].

In this paper we use the symmetric properties of the domain of our boundary value problem. This allows us to reduce the problem to a system of functional equations on multiply connected circular domains. Further, we generalize the method proposed in the scalar case in [25] to vector-matrix problems and construct solution of this problem. The final form of the solution involves the Christoffel-Schwarz transformation of the half-plane onto a circular polygon which contains undetermined parameters satisfying some equations. Solution of the functional equations is obtained in the form of the matrix Poincare series.

\section{2. $\mathbb{C}$-linear conjugation problem on a finite number of real intervals}

In this section we consider the $\mathbb{C}$-linear conjugation problem (or Riemann boundary value problem) on $n$ intervals of real line with respect to analytic $N$-vectors. We reformulate this problem in the form of the Riemann-Hilbert boundary value problem with respect to analytic $2 N$-vectors with suitably chosen matrix coefficient.

Let $-\infty<A_{0}<A_{1}<\ldots<A_{2 n-2}<A_{2 n-1}<\infty$ be given points on the real line. Denote by $L_{k}=\left(A_{2 k-2}, A_{2 k-1}\right)$ the open intervals on the real line $\mathbb{R}$ with the end points $A_{2 k-2}, A_{2 k-1}, L=\bigcup_{k=1}^{n} \operatorname{cl} L_{k}, \widetilde{D}=\mathbb{C} \backslash L$, where cl $L_{k}$ is the closure of $L_{k}$. Let a constant nonsingular $N \times N$ matrix $G_{k}$ satisfying the condition $G_{k}^{2}=E$ be given on each interval $L_{k}$.

Consider the following $\mathbb{C}$-linear conjugation problem: Find a vector-function $\Phi(z)$, which is analytic in $\widetilde{D}$, continuously extended up to both sides of $L_{k}$, bounded at each point $A_{k}$, having at most polynomial growth of order $(n-1)$ at infinity, and satisfying the following boundary conditions

$$
\Phi^{+}(t)=G_{k} \Phi^{-}(t), \quad t \in L_{k} .
$$

By defining the vector-function in the upper half-plane $\mathbb{C}^{+}$

$$
\phi(z) \equiv-i\left(\frac{\Phi(z)}{\Phi(\bar{z})}\right)
$$

we can reformulate boundary condition (2.1) in the form of the boundary condition of the Riemann-Hilbert boundary value problem with respect to $2 N$-vector $\phi(z)$ :

$$
\operatorname{Re}\left\{\overline{\lambda_{k}} \phi(t)\right\}=0, \quad t \in L_{k}, k=1, \ldots, n,
$$


where the matrices $\lambda_{k}$ satisfy the relation

$$
\overline{\lambda_{k}} g_{k}=\lambda_{k}
$$

with $g_{k}:=\left(\begin{array}{cc}\frac{0}{G_{k}^{-1}} & 0\end{array}\right)$. In order to see that the above reduction from problem $(2.1)$ to $(2.3)$ is correct we do some calculation. First, transform the expression $\overline{\lambda_{k}} \phi(t)$ on the upper side of $k$-th interval:

$$
\overline{\lambda_{k}} \phi(t)=-i \overline{\lambda_{k}}\left(\frac{\Phi^{+}(t)}{\Phi^{-}(t)}\right) .
$$

It follows from the boundary condition (2.1) that the right-hand side of the latter is equal

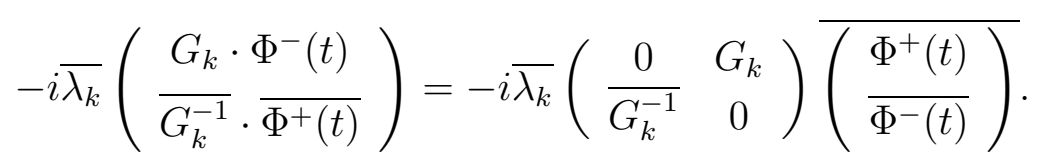

By (2.4) it is equal to $-\lambda_{k} \overline{\phi(t)}$. Therefore, $\overline{\lambda_{k}} \phi(t) \equiv-\lambda_{k} \overline{\phi(t)}, t \in L_{k}$, hence the boundary condition (2.3) follows. In the same manner one can check, that vector function (2.2) defined in the lower half-plane satisfies also boundary condition (2.3) on the lower side of the interval with the matrix $\lambda_{k}$ chosen according to relation (2.4) providing the validity of boundary condition (2.1).

Since the matrix $g_{k}$ satisfies the condition $g_{k}^{-1}=\overline{g_{k}}$ the problems $(2.1)$ and (2.3) are equivalent in the following sense. Any solution $\Phi(z)$ to problem (2.1) determines via representation (2.2) the solution to problem (2.3). Vice versa, any solution to problem (2.3) satisfies the relation $\left(\phi_{1}(z), \ldots, \phi_{N}(z)\right)^{T} \equiv$ $\left(\overline{\phi_{N+1}(\bar{z})}, \ldots, \overline{\phi_{2 N}(\bar{z})}\right)^{T}$. Therefore, the first $N$ components of the solution $\phi(z)$ to problem (2.3) constitute the solution to problem (2.1).

The choice of the matrix $\lambda_{k}$ satisfying relation (2.4) is in fact not unique. One can take $\lambda_{k}$ as a square root of the matrix $g_{k}$, i.e.,

$$
\lambda_{k}^{2}=g_{k}
$$

Then it follows from (2.4) that such matrix $\lambda_{k}$ does satisfy the following relation

$$
\lambda_{k} \overline{\lambda_{k}}=E,
$$

where $E$ is a unit $2 N \times 2 N$-matrix.

\section{Reduction to the problem for a multiply connected circular domain}

By using the Christoffel-Schwarz formula for the considered domain problem (2.3) is reduced here to the Riemann-Hilbert problem for a multiply connected circular domain with the same unknown $2 N$ analytic vector. This problem will be studied then in the next sections. 
Consider $2 n$ points $B_{j}(j=0,1, \ldots, 2 n-1)$ on the real axis of the complex plane $\zeta$. Here we assume that $B_{j_{0}+1}<B_{j_{0}+2}<\ldots<B_{2 n-1}<B_{0}<B_{1}<\ldots<$ $B_{j_{0}}$ for some $j_{0}$. Consider the segments $\left(B_{j}, B_{j+1}\right)$, where $j=2 k-1,2 k+1, \ldots$ $(\bmod 2 n)$ (including $\left(B_{j_{0}}, B_{j_{0}+1}\right)=\left(B_{j_{0}},+\infty\right) \cup\left(-\infty, B_{j_{0}+1}\right)$ if $j_{0}$ is odd), and the semi-circles $\curvearrowright\left(B_{j}, B_{j+1}\right):=\left\{\zeta \in \mathbb{C}: \operatorname{Im} \zeta \geq 0,\left|\zeta-d_{j}\right|=R_{j}\right\}$, where $j=2 k-2,2 k, \ldots(\bmod 2 n), d_{j}=\frac{B_{j+1}+B_{j}}{2}, R_{j}=\frac{B_{j+1}-B_{j}}{2}$ for $j \neq j_{0}$ and $R_{j_{0}}=\frac{B_{j_{0}}-B_{j_{0}+1}}{2}$ if $j_{0}$ is even. Therefore, we obtain the domain $D^{\prime}$ bounded by the segments $\left(B_{2 k-1}, B_{2 k}\right)$ and semi-circles $\curvearrowright\left(B_{2 k-2}, B_{2 k-1}\right)$, where the semicircle $\curvearrowright\left(B_{j_{0}}, B_{j_{0}+1}\right)$ is exterior for $D^{\prime}$ if $j_{0}$ is even.

We construct a conformal mapping $\zeta=f(z)$ of the upper half-plane $\operatorname{Im} z>$ 0 onto $D^{\prime}$ in such a way that $f\left(A_{l}\right)=B_{l}(l=0,1, \ldots, 2 n-1)$. Here the given points $A_{l}$ lie on the real axis (see Sec. 2), and $B_{l}$ are undetermined real constants. The function $f(z)$ can be represented in the form [26]

$$
f(z)=\frac{y_{1}(z)}{y_{2}(z)}+c
$$

where $y_{1}(z), y_{2}(z)$ are two fixed linearly independent solutions of the ordinary differential equation

$$
y^{\prime \prime}(z)+\sum_{l=0}^{2 n-1}\left(\frac{1-\alpha_{l}^{2}}{4\left(z-d_{l}\right)^{2}}+\frac{1}{2} \frac{\beta_{l}}{z-d_{l}}\right) y(z)=0
$$

$c$ is a constant. Here $\beta_{l}(l=0,1, \ldots, 2 n-1)$ are undetermined real constants satisfying the relations

$$
\sum_{l=0}^{2 n-1} \beta_{l}=0, \quad \sum_{l=0}^{2 n-1}\left(2 d_{l} \beta_{l}+1-\alpha_{l}^{2}\right)=0, \quad \sum_{l=0}^{2 n-1}\left(d_{l}^{2} \beta_{l}+d_{l}\left(1-\alpha_{l}^{2}\right)\right)=0
$$

$\pi \alpha_{l}(l=0,1, \ldots, 2 n-1)$ are angles of the polygon $D^{\prime}$ at the vertices $B_{l}$, respectively. In our case all $\alpha_{l}=\frac{1}{2}$, hence (3.2) becomes

$$
y^{\prime \prime}(z)+\frac{1}{2} R(z) y(z)=0
$$

where $R(z)=\sum_{l=0}^{2 n-1}\left(\frac{3}{8} \frac{1}{\left(z-d_{l}\right)^{2}}+\frac{\beta_{l}}{z-d_{l}}\right)$. The conditions (3.3) become

$$
\sum_{l=0}^{2 n-1} \beta_{l}=0, \quad \sum_{l=0}^{2 n-1} d_{l} \beta_{l}=-\frac{3}{8}, \quad \sum_{l=0}^{2 n-1} d_{l}^{2} \beta_{l}=-\frac{3}{4} \sum_{l=0}^{2 n-1} d_{l}
$$

Moreover, the Schwarzian derivative is equal $\left(\frac{f^{\prime \prime}(z)}{f^{\prime}(z)}\right)^{\prime}-\frac{1}{2}\left(\frac{f^{\prime \prime}(z)}{f^{\prime}(z)}\right)^{2}=R(z)$.

It is worth to note that in our case the $d_{l}$ are given, contrary to the classical problem of the Christoffel-Schwarz transformation. Various values of the 
real constants $\beta_{0}, \beta_{1}, \ldots, \beta_{2 n-1}$, Re $c$, Im $c$ correspond to various circular polygons $B_{0} B_{1} \ldots B_{2 n-1}$ with angles $\frac{\pi}{2}$ on the plane $\zeta$ (generally speaking on the Riemann surface of the function $\zeta=f(z)$ ). We are now looking for additional restrictions on the undetermined parameters which yield the required polygon $B_{0} B_{1} \ldots B_{2 n-1}$ described at the beginning of the section. Existence of the conformal mapping $\zeta=f(z)$ follows from the existence of the conformal mapping of the multiply connected domain $\mathbb{C} \backslash \bigcup_{k=1}^{n} L_{k}$ onto a circular domain. Moreover, the symmetry line on the plane $z$ has to be transformed onto the symmetry line of the domain $D^{\prime}$. Therefore, the real axis on the plane $z$ is transformed onto the real axis on the plane $\zeta$.

We assume that the segments $\left(A_{j}, A_{j+1}\right)$ with odd $j=2 k-1$, i.e., the segments $\left(A_{2 k-1}, A_{2 k}\right)$, are transformed onto the segments $\left(B_{j}, B_{j+1}\right)$. Then the segments $L_{k}=\left(A_{2 k-2}, A_{2 k-1}\right)$ with even $j=2 k$ are transformed onto the semi-circles $\curvearrowright\left(B_{2 k-2}, B_{2 k-1}\right)$. In particular, the infinite point $z=\infty$ belonging to the segment $\left(A_{2 n-1}, A_{0}\right)$, is transformed to a point $\zeta_{\infty} \in\left(B_{2 n-1}, B_{0}\right) \in \mathbb{R}$. Then, using (3.1) we have

$$
\operatorname{Im} c=-\operatorname{Im} \frac{y_{1}(\infty)}{y_{2}(\infty)} .
$$

We assume that $\operatorname{Re} c=0$, since the term $\operatorname{Re} c$ in (3.1) corresponds to the shift of the plane $\zeta$ parallel to the real axis.

The conditions

$$
\operatorname{Im} f\left(A_{l}\right)=0, \quad l=0,1, \ldots, 2 n-1,
$$

mean that all points $B_{l}$ lie on the real line. However, two of $2 n$ conditions (3.7) are redundant. First, we have $f\left(A_{0}\right)=B_{0} \in \mathbb{R}$, due to (3.6), i.e., the segment $\left(B_{2 n-1}, \zeta_{\infty}\right)$ lies on the real axis and can be continued to the point $B_{0}$ if only $B_{0} \in \mathbb{R}$. Second, if $B_{j_{0}+1}<B_{j_{0}+2}<\ldots<B_{2 n-1}<B_{0}<B_{1}<\ldots<B_{j_{0}-1}$, and all these $B_{j}$ are real, then $B_{j_{0}}$ is real too. It is so, since the real axis is tangent to a boundary arc $\left(B_{j_{0}-1}, B_{j_{0}}\right)$ at $B_{j_{0}-1}$ and it is perpendicular to the semi-circle $\curvearrowright\left(B_{j_{0}}, B_{j_{0}+1}\right)$ whose diameter lies on the real axis. Hence, this arc $\left(B_{j_{0}-1}, B_{j_{0}}\right)$ can be only a segment of the real axis. Thus, instead of $(3.7)$ it is sufficient to take the conditions

$$
\operatorname{Im} f\left(A_{l}\right)=0, \quad l=1,2, \ldots, 2 n-1 ; l \neq j_{0} .
$$

Ultimately we have $2 n+1$ undetermined real constants $\beta_{0}, \beta_{1}, \ldots, \beta_{2 n-1}, \operatorname{Im} c$ satisfying $2 n+2$ conditions (3.5), (3.6), (3.8). Our conjecture is that (3.8) contains only one redundant condition, but we cannot prove it yet.

Equation (3.4) is the classical Fuchsian ordinary differential equation with singular points. The method of the series expansion can be successfully applied 
to construct its fundamental solutions $y_{1}(z)$ and $y_{2}(z)[18]$. The functions $y_{1}(z)$ and $y_{2}(z)$ depend on the parameters $\beta_{l}(l=0,1, \ldots, 2 n-1)$. Three of these parameters, e.g., $\beta_{2 n-3}, \beta_{2 n-2}, \beta_{2 n-1}$, can be expressed from linear relations (3.5) via $\beta_{l}(l=0,1, \ldots, 2 n-4)$. Then the conditions (3.8) yield the non-linear equations

$$
\operatorname{Im}\left(\frac{y_{1}\left(A_{l}\right)}{y_{2}\left(A_{l}\right)}-\frac{y_{1}(\infty)}{y_{2}(\infty)}\right)=0, \quad l=1,2, \ldots, 2 n-1 ; l \neq j_{0},
$$

with respect to real unknowns $\beta_{l}(l=0,1, \ldots, 2 n-4)$. Each solution $\beta_{l}(l=$ $0,1, \ldots, 2 n-4)$ of $(3.9)$ determines a conformal mapping $\zeta=f(z)$ of $\mathbb{C}^{+}$onto a domain $D^{\prime}$ of the required type. Moreover, there exists at least one solution to the system (3.9), since there exists the required conformal mapping.

Further, it is convenient to deal with an unbounded domain $D^{\prime}$. For this (in the case when $j_{0}$ is even) we map $D^{\prime}$ onto the half of a circular domain $\mathbb{D} \ni \infty$ by the mapping $\zeta \mapsto \frac{R_{j_{0}}^{2}}{\zeta-d_{j_{0}}}$. Next, we construct the conformal mapping

$$
\omega(z)=\frac{R_{j_{0}}^{2}}{f(z)-d_{j_{0}}}
$$

and reduce the problem (2.3) to the following equivalent form:

$$
\operatorname{Re}\left\{\overline{\lambda_{k}} \varphi(\tau)\right\}=0, \quad \tau \in L_{k}^{+}, k=1, \ldots, n,
$$

with respect to the function $\varphi$ analytic in $\mathbb{D}^{+}$determined by the relation $\phi=$ $\varphi \circ \omega$. Here $L_{k}^{+}=\left\{\tau \in \mathbb{C}^{+}:\left|\tau-a_{k}\right|=r_{k}\right\}$.

Using the analytic continuation of $\varphi(\zeta)$ into the lower half-plane $\mathbb{C}^{-}$by $(2.2)$ and by the symmetry property of the conformal mapping $\zeta=f(z)$ we obtain that

$$
\varphi(\tau)+\overline{g_{k}^{-1} \varphi(\tau)}=0, \quad \tau \in L_{k}^{-}=\left\{\tau \in \mathbb{C}^{-}:\left|\tau-a_{k}\right|=r_{k}\right\}
$$

If we additionally impose the condition (1.3), then $\overline{g_{k}^{-1}}=g_{k}$ and (3.11), (3.12) yield the Riemann-Hilbert problems for multiply connected circular domain

$$
\operatorname{Re}\left\{\overline{\lambda_{k}} \varphi(\tau)\right\}=0, \quad\left|\tau-a_{k}\right|=r_{k}, k=1, \ldots, n .
$$

The condition (1.3) is necessary to justify the reduction of the $\mathbb{C}$-linear problem to the Riemann-Hilbert problem. However, it is not used in the solution to the problem (3.13). Therefore, below we do not use (1.3) and we solve (3.13) with general $\lambda_{k}$ satisfying the condition (2.6) which is natural for this type of problem. 


\section{Solution of the Riemann-Hilbert problem for a multiply connected circular domain}

4.1. Schottky groups and spaces for solutions. Let us briefly outline some notation and facts from the theory of functional equations in complex domains following [25].

Consider the family of disjoint discs $\mathbb{D}_{k}=\left\{\zeta \in \mathbb{C}:\left|\zeta-a_{k}\right|<r_{k}\right\}, k=$ $1, \ldots, n, \mathbb{D} \equiv \widehat{\mathbb{C}} \backslash \bigcup_{k=1}^{n} \operatorname{cl} \mathbb{D}_{k}$. Denote also $\mathbb{T}_{k} \equiv \partial \mathbb{D}_{k}, k=1, \ldots, n$. Introduce transformations $z_{\left(k_{m}, k_{m-1}, \ldots, k_{1}\right)}^{*} \equiv\left(z_{\left(k_{m-1}, \ldots, k_{1}\right)}^{*}\right)_{\left(k_{m}\right)}^{*}$, where $z_{(k)}^{*} \equiv \frac{r_{k}^{2}}{\overline{z-a_{k}}}+a_{k}$ is the symmetry mapping with respect to $k$-th circle $\mathbb{T}_{k}$. If in the sequence $k_{m}, k_{m-1}, \ldots, k_{1}$ no two consequent numbers are equal then $m$ is called the level of the transformation. All such transformations generate the so called Schottky group of symmetries $\mathcal{K}$ (for shortness we denote the elements of this group by $\gamma_{j}$ in accordance to certain fixed order relevant to the increase of their level) (see [14]). The collection of all $\gamma_{j}$ of even level forms the subgroup $\mathcal{G}$, and those of odd level form the conjugate class $\mathcal{F}=\mathcal{K} \backslash \mathcal{G}$. Elements of $\mathcal{G}$ are simply Möbius transformations, i.e., they have the form $\gamma_{j}(\zeta)=\frac{\hat{a}_{j} z+\hat{b}_{j}}{\hat{c}_{j} z+\hat{d}_{j}}, \hat{a}_{j} \hat{d}_{j}-\hat{b}_{j} \hat{c}_{j}=1$. Elements of $\mathcal{F}$ are anti-Möbius transformations, i.e., Möbius with respect to $\bar{\zeta}$.

Introduce the Banach space of vector-functions $\mathbf{f}(\zeta)=\left(f_{1}(\zeta), \ldots, f_{M}(\zeta)\right)^{T}$ $\in \mathcal{C}\left(\bigcup_{k=1}^{n} \mathbb{T}_{k}\right)$ with the norm

$$
\|\mathbf{f}\|=\sup _{k=1, \ldots, n} \sup _{\zeta \in \mathbb{T}_{k}}\left(\left|f_{1}(\zeta)\right|^{2}+\cdots+\left|f_{M}(\zeta)\right|^{2}\right)^{\frac{1}{2}}
$$

and its closed subspace $\mathcal{C}_{\mathcal{A}}\left(\bigcup_{k=1}^{n} \mathbb{T}_{k}\right)$ of all vector-functions admitting an analytic continuation into all domains $\mathbb{D}_{k}, k=1, \ldots, n$, endowed with the norm (4.1). From the maximum principle it follows that the space $\mathcal{C}_{\mathcal{A}}\left(\bigcup_{k=1}^{n} \mathbb{T}_{k}\right)$ coincides with the space $\mathcal{C}_{\mathcal{A}}\left(\bigcup_{k=1}^{n} \mathrm{cl} \mathbb{D}_{k}\right)$ of all vector functions analytic in the domains $\mathbb{D}_{k}, k=1, \ldots, n$ and continuous up to their boundaries $\mathbb{T}_{k}, k=1, \ldots, n$. Hereafter we fix a point $w \in \mathbb{D} \backslash\{\infty\}$.

4.2. Reduction of the problem to functional equations. Following [25, p. 161] we rewrite boundary conditions (3.11) in the following form:

$$
\overline{\lambda_{k}} \varphi(\tau)=\varphi_{k}(\tau)-\overline{\varphi_{k}(\tau)}, \tau \in \mathbb{T}_{k} \equiv\left\{\tau \in \mathbb{C}:\left|\tau-a_{k}\right|=r_{k}\right\}, k=1, \ldots, n,
$$

where auxiliary vector-functions $\varphi_{k}(\zeta)$ are analytic in the discs $\mathbb{D}_{k} \equiv\{\zeta \in \mathbb{C}$ : $\left.\left|\zeta-a_{k}\right|<r_{k}\right\}$, continuous in $\mathrm{cl}_{\mathbb{D}_{k}}$. The unknown vector-function $\varphi$ has to be analytic in the outer multiply connected circular domain $\mathbb{D} \equiv \mathbb{C} \backslash \sum_{k=1}^{n} \mathrm{cl} \mathbb{D}_{k}$ having polynomial growth of order $(n-1)$ at infinity.

Let us introduce the following vector-function

$$
\Omega(\zeta) \equiv \begin{cases}\lambda_{k} \varphi_{k}(\zeta)+\sum_{m \neq k} \lambda_{m} \overline{\varphi_{m}\left(\zeta_{(m)}^{*}\right)}, & \zeta \in \operatorname{cl} \mathbb{D}_{k} \\ \varphi(\zeta)+\sum_{m=1}^{n} \lambda_{m} \overline{\varphi_{m}\left(\zeta_{(m)}^{*}\right)}, & \zeta \in \mathbb{D} .\end{cases}
$$


Since on all circles $\mathbb{T}_{k}$ we have $\Omega^{+}(\tau)=\Omega^{-}(\tau), \tau \in \mathbb{T}_{k}, k=1, \ldots, n$, then analytic continuation principle and the Liouville theorem imply that $\Omega$ is simply a polynomial vector, i.e.,

$$
\Omega(\zeta)=\hat{\mathbf{P}}_{n-1}(\zeta) \equiv\left(P_{n-1}^{(1)}(\zeta), \ldots, P_{n-1}^{(2 N)}(\zeta)\right)^{T} .
$$

By using definition of the function $\Omega$ and the relation $\lambda_{k} \overline{\lambda_{k}}=E$ we arrive then to system of functional equations on each closed disc $\mathbb{D}_{k}(k=1, \ldots, n)$

$$
\varphi_{k}(\zeta)=-\overline{\lambda_{k}} \sum_{m \neq k} \lambda_{m} \overline{\varphi_{m}\left(\zeta_{(m)}^{*}\right)}+\overline{\lambda_{k}} \hat{\mathbf{P}}_{n-1}(\zeta), \quad \zeta \in \mathrm{cl} \mathbb{D}_{k}
$$

4.3. Solution to functional equations. Introduce the vector functions $\psi_{k}(\zeta)$ in such a way that

$$
\varphi_{k}(\zeta)=\sum_{j=0}^{\infty} \alpha_{j k}\left(\zeta-a_{k}\right)^{j}=\mathbf{P}_{M-1, k}(\zeta)+\left(\frac{\zeta-a_{k}}{r_{k}}\right)^{M} \psi_{k}(\zeta),
$$

with the polynomial vector

$$
\mathbf{P}_{M-1, k}(\zeta)=\sum_{j=0}^{M-1} \alpha_{j k}\left(\zeta-a_{k}\right)^{j}
$$

Let $\psi(\zeta)=\psi_{k}(\zeta), \zeta \in \operatorname{cl} \mathbb{D}_{k}$. Substituting (4.4) into (4.3) we arrive at the functional equations with respect to $\psi \in \mathcal{C}_{\mathcal{A}}\left(\bigcup_{k=1}^{n} \mathbb{T}_{k}\right)$

$$
\begin{aligned}
\psi_{k}(\zeta)= & -\overline{\lambda_{k}} \sum_{m \neq k} \lambda_{m}\left(\frac{r_{k} r_{m}}{\left(\zeta-a_{k}\right)\left(\zeta-a_{m}\right)}\right)^{M} \overline{\psi_{m}\left(\zeta_{(m)}^{*}\right)} \\
& +\left(\frac{r_{k}}{\zeta-a_{k}}\right)^{M} h(\zeta), \quad \zeta \in \mathrm{cl} \mathbb{D}_{k},
\end{aligned}
$$

where

$$
h(\zeta)=\overline{\lambda_{k}} \hat{\mathbf{P}}_{n-1}(\zeta)-\mathbf{P}_{M-1, k}(\zeta)-\overline{\lambda_{k}} \sum_{m \neq k} \lambda_{m} \overline{\mathbf{P}_{M-1, m}\left(\zeta_{(m)}^{*}\right)}
$$

By Cauchy's formula,

$$
\psi_{m}\left(\zeta_{(m)}^{*}\right)=\frac{1}{2 \pi i} \int_{\mathbb{T}_{m}} \frac{\psi_{m}(\tau)}{\tau-\zeta_{(m)}^{*}} d \tau, \quad \zeta \in \mathrm{cl} \mathbb{D}_{k}(m \neq k) .
$$

Substituting (4.8) into (4.6) we arrive at the integral equations

$$
\begin{aligned}
\Psi_{k}(\zeta)= & -\overline{\lambda_{k}} \sum_{m \neq k} \lambda_{m}\left(\frac{r_{k} r_{m}}{\left(\zeta-a_{k}\right)\left(\zeta-a_{m}\right)}\right)^{M} \frac{1}{2 \pi i} \int_{\mathbb{T}_{m}} \frac{\Psi_{m}(\tau)}{\tau-\zeta_{(m)}^{*}} d \tau \\
& +\left(\frac{r_{k}}{\zeta-a_{k}}\right)^{M} h(\zeta), \quad \zeta \in \mathrm{cl} \mathbb{T}_{k}(k=1,2, \ldots, n) .
\end{aligned}
$$


Instead of $\psi_{k}(\zeta), \Psi_{k}(\zeta)$ is written. Formally equations (4.9) can be considered as Fredholm's integral equations with respect to $\Psi \in \mathcal{C}\left(\bigcup_{k=1}^{n} \mathbb{T}_{k}\right)$. First, equations (4.9) will be solved in $\mathcal{C}\left(\bigcup_{k=1}^{n} \mathbb{T}_{k}\right)$. Then it will be checked, that continuous vector-function $\Psi$ can be analytically continued in the corresponding domains, i.e., $\Psi \in \mathcal{C}_{\mathcal{A}}\left(\bigcup_{k=1}^{n} \mathbb{T}_{k}\right)$. If so then $\Psi_{k}(\zeta)=\psi_{k}(\zeta)$ satisfy the functional equations (4.6).

Let us rewrite (4.9) in the following compact form:

$$
\Psi=\mathbf{A} \Psi+\hat{h}
$$

where the linear operator $\mathbf{A}$ and $\hat{h}$ are determined by the right-hand side of the system (4.9). As it was shown in [25, p. 148] the operator $\mathbf{A}$ is compact in $\mathcal{C}\left(\bigcup_{k=1}^{n} \mathbb{T}_{k}\right)$. It is easily seen that

$$
\left|\frac{r_{k} r_{m}}{\left(\zeta-a_{k}\right)\left(\zeta-a_{m}\right)}\right| \leq\left|\frac{r_{m}}{a_{k}-a_{m}}\right| \leq q<1, \quad \zeta \in \operatorname{cl} \mathbb{T}_{k}(m \neq k) .
$$

Therefore, for sufficiently large $M$ the norm of the operator $\mathbf{A}$ is less than unity. Then equation (4.10) can be solved by the method of successive approximations which yield the solution in the form of the series

$$
\Psi=\sum_{j=0}^{\infty} \mathbf{A}^{j} \hat{h}
$$

In order to write (4.11) in expanded form we note that the right hand part of (4.9) is analytic in all $\left|\zeta-a_{k}\right|<r_{k}$ except $a_{k}$ where it has a pole of order $M$. Then (4.9) implies that $\Psi_{k}(\zeta)$ also belongs to the same class and

$$
\frac{1}{2 \pi i} \int_{\mathbb{T}_{m}} \frac{\Psi_{m}(\tau)}{\tau-\zeta_{(m)}^{*}} d \tau=\Psi_{m}\left(\zeta_{(m)}^{*}\right)+\operatorname{res}_{a_{m}} \frac{\Psi_{m}(\tau)}{\tau-\zeta_{(m)}^{*}} .
$$

Calculation of the residium yields

$$
\frac{1}{2 \pi i} \int_{\mathbb{T}_{m}} \frac{\Psi_{m}(\tau)}{\tau-\zeta_{(m)}^{*}} d \tau=\Psi_{m}\left(\zeta_{(m)}^{*}\right)-\left\{\Psi_{m}\right\}\left(\zeta_{(m)}^{*}\right),
$$

where the principal part $\left\{\Psi_{m}\right\}$ of $\Psi_{m}$ is introduced. More precisely, $\left\{\Psi_{m}\right\}(\zeta)=$ $\frac{\psi_{-M}}{\left(\zeta-a_{m}\right)^{M}}+\cdots+\frac{\psi_{-1}}{\zeta-a_{m}}$, where $\Psi_{m}(\zeta)$ is presented via its Laurent's series

$$
\Psi_{m}(\zeta)=\frac{\psi_{-M}}{\left(\zeta-a_{m}\right)^{M}}+\cdots+\frac{\psi_{-1}}{\zeta-a_{m}}+\psi_{0}+\psi_{1}\left(\zeta-a_{m}\right)+\cdots
$$

The iteration scheme corresponding to the series (4.11) can be written in the following form. The zero-th term has the form

$$
\left(\frac{\zeta-a_{k}}{r_{k}}\right)^{M} \Psi_{k}^{(0)}(\zeta)=\overline{\lambda_{k}} h(\zeta)
$$


The iteration formula for the term $p+1$ has the form

$$
\begin{aligned}
\left(\frac{\zeta-a_{k}}{r_{k}}\right)^{M}[ & \left.\Psi_{k}^{(p+1)}(\zeta)-\left\{\Psi_{k}^{(p+1)}\right\}(\zeta)\right] \\
= & -\overline{\lambda_{k}} \sum_{m \neq k} \lambda_{m}\left(\frac{r_{m}}{\zeta-a_{m}}\right)^{M}\left[\overline{\Psi_{m}^{(p)}\left(\zeta_{(m)}^{*}\right)}-\overline{\left\{\Psi_{m}^{(p)}\right\}\left(\zeta_{(m)}^{*}\right)}\right] \\
& +h(\zeta)-\lambda_{k}\left(\frac{\zeta-a_{k}}{r_{k}}\right)^{M}\left\{\Psi_{k}^{(p+1)}\right\}(\zeta) .
\end{aligned}
$$

Applying the iteration scheme (4.12)-(4.13) we arrive at the formula

$$
\Psi_{k}(\zeta)=\left(\frac{r_{k}}{\zeta-a_{k}}\right)^{M} \mathbf{A}_{k} h(\zeta)+\left\{\Psi_{k}\right\}(\zeta),
$$

where the operator $\mathbf{A}_{k}$ is introduced as follows

$$
\begin{aligned}
\mathbf{A}_{k} h(\zeta) \equiv & \overline{\lambda_{k}} \sum_{m=1}^{\infty}(-1)^{m} \sum_{k_{1} \neq k} \sum_{k_{2} \neq k_{1}} \ldots \sum_{k_{m} \neq k_{m-1}} \lambda_{k_{1}} \overline{\lambda_{k_{2}}} \cdot \ldots \cdot\left(\mathbf{C}^{m-2} \lambda_{k_{m-1}}\right) \\
& \cdot\left(\mathbf{C}^{m-1} \lambda_{k_{m}}\right) \cdot \mathbf{C}^{m}\left[h\left(\zeta_{\left(k_{m}, \ldots, k_{1}\right)}^{*}\right)-\left\{h\left(\zeta_{\left(k_{m}, \ldots, k_{1}\right)}^{*}\right)\right\}\right]+h(\zeta) .
\end{aligned}
$$

Here $\mathbf{C}$ is the operator of the complex conjugation. The principal part $\left\{\Psi_{k}\right\}(\zeta)$ stays undetermined. The operator $\mathbf{A}_{k}$ is analogous to that proposed in the scalar case in $[25$, p. 162]. It realizes the method of the successive approximations in the considered case.

It follows from (4.14) that $\Psi_{k}(\zeta)$ is analytical at $a_{k}$ (in other words $\Psi_{k}(\zeta)=$ $\left.\psi_{k}(\zeta)\right)$ if and only if $\mathbf{A}_{k} h(\zeta)$ has zero of order $M$ at $a_{k}$. This condition can be written in the form

$$
\int_{\mathbb{T}_{k}} \mathbf{A}_{k} h(\zeta)\left(\zeta-a_{k}\right)^{-j} d \zeta=0, \quad j=1,2, \ldots, M
$$

Therefore, the functional equations (4.6) are solvable if and only if the conditions (4.16) are fulfilled. If they are fulfilled the solution has the form $\psi_{k}(\zeta)=\left(\frac{r_{k}}{\zeta-a_{k}}\right)^{M} \mathbf{A}_{k} h(\zeta)$. It follows from (4.7) and (4.15) that that the conditions (4.16) are linear algebraic equations with respect to the coefficients of the vector polynomials $\hat{\mathbf{P}}_{n-1}(\zeta)$ and $\mathbf{P}_{M-1, k}(\zeta)$. Some of them can be stay undetermined after satisfaction of the system (4.16). The vector functions $\varphi_{k}(\zeta)$ are found from (4.4)

$$
\varphi_{k}(\zeta)=\mathbf{P}_{M-1, k}(\zeta)+\mathbf{A}_{k} h(\zeta)
$$

Finally, from the definition of the function $\Omega$ it follows that the general solution to the problem (3.11) is presented in the form

$$
\varphi(\zeta)=\hat{\mathbf{P}}_{n-1}(\zeta)-\sum_{m=1}^{n} \lambda_{m} \overline{\varphi_{m}\left(\zeta_{(m)}^{*}\right)}, \quad \zeta \in \mathbb{D},
$$


The general solution to problem (2.3) is given by the formula

$$
\phi(z) \equiv \varphi(\omega(z)), \quad z \in \widetilde{D}
$$

where $\omega$ is the conformal mapping of $\widetilde{D}$ onto $\mathbb{D}$ constructed in Section 3 via formulas (3.10) and (3.1). The first $N$ components of the vector $i \phi(z)$ form the solution $\Phi(z)$ of the starting $\mathbb{C}$-linear conjugation problem $(2.1)$ with piecewise constant matrix coefficient.

Note added in proof. In the scalar case (when the unknown functions take their values in $\mathbb{C}$ ), the system of functional equations (4.3) with constants $\lambda_{k}$ $\left(\left|\lambda_{k}\right|=1\right)$ has always a unique solution. This solution can be found by successive approximations [25]. If we assume that the matrices $\lambda_{k}$ have such a structure that successive approximations converge in the vector-matrix case, then $M=0$. Hence the vector polynomial (4.5) vanishes and representation (4.17) becomes $\varphi_{k}(\zeta)=\mathbf{A}_{k} h(\zeta)$

In general vector-matrix case, successive approximations applied to functional equations (4.3) can be divergent. Therefore, the method fit for the scalar case have to be modified as it is done in Subsec. 4.3. For better presentation of the modified scheme we add auxiliary notations and facts in Subsec. 4.1.

Acknowledgments. S. R. thanks the Belarusian Fund for Fundamental Scientific Research for partial support of this work.

The authors are grateful to Prof. Frank-Olme Speck for valuable discussion of the problems during our visits supported by Centro de Matemática e Aplicações of Instituto Superior Técnico, Lisboa.

We thank the referees for their useful comments and corrections.

\section{References}

[1] Ablowitz, M. J. and Fokas, A. S., Complex Variables: Introduction and Applications (2nd ed.). Cambridge: Cambridge University Press 2003.

[2] Aptekarev, A. I. and Van Assche, W., Scalar and matrix Riemann-Hilbert approach to the strong asymptotics of Padé approximants and complex orthogonal polynomials with varying weight. J. Approx. Theory 129 (2004), 129 - 166.

[3] Arnold, V. I. and Il'yashenko, Yu. S., Ordinary differential equations (in Russian). In: Current Problems in Mathematics. Fundamental Directions 1. Moscow: Akad. Nauk SSSR, Vsesoyuz. Inst. Nauchn. i Tekhn. Inform. (VINITI) 1985, pp. 7 - 149.

[4] Bishop, C. J., Böttcher, A., Karlovich, Yu. I. and Spitkovsky, I. M., Local spectra and index of singular integral operators with piecewise continuous coefficients on composed curves. Math. Nachr. 206 (1999), 5 - 83. 
[5] Bolibrukh, A. A., The Riemann-Hilbert problem (in Russian). Uspekhi Mat. Nauk 45 (1990)(2), 3 - 47; transl. in: Russian Math. Surveys 45 (1990)(2/272), $1-58$.

[6] Bolibrukh, A. A., Regular systems whose monodromies differ from monodromies of Fuchsian systems. J. Math. Sci. 78 (1996)(5), 530 - 541.

[7] Bolibrukh, A. A., Its, A. R. and Kapaev, A. A., On the Riemann-HilbertBirkhoff inverse monodromy problem and the Painlevé equations (in Russian). Algebra i Analiz 16 (2004)(1), 121 - 162; transl. in: St. Petersburg Math. J. 16 (2004)(1), $105-142$.

[8] Böttcher, A., Karlovich, Yu. I. and Spitkovsky, I. M., Convolution Operators and Factorization of Almost Periodic Matrix Functions. Oper. Theory: Adv. Appl. 131. Basel: Birkhäuser 2002.

[9] Clancey, K. and Gohberg, I., Factorization of Matrix Functions and Singular Integral Operators. Oper. Theory: Adv. Appl. 3. Basel: Birkhäuser 1981.

[10] Conceição, A. C., Kravchenko, V. G. and Teixeira, F. S., Factorization of some classes of matrix functions and the resolvents of a Hankel operator. In: Factorization, Singular Operators and Related Problems (Funchal 2002; eds.: S. G. Samko et al.) Dordrecht: Kluwer 2003, pp. $101-110$.

[11] Ehrhardt, T. and Spitkovskiǔ, I. M., Factorization of piecewise constant matrixvalued functions, and systems of linear differential equations (in Russian). Algebra i Analiz 13 (2001)(6), 56 - 123; transl. in: St. Petersburg Math. J. 13 (2001)(6), $939-991$.

[12] Ehrhardt, T. and Speck, F.-O., Transformation techniques towards the factorization of $2 \times 2$ matrix functions. Linear Algebra Appl. 353 (2002)(1-3), 53 - 90 .

[13] Fokas, A. S., Its, A. R. and Kitaev, A. V., The isomonodromy approach to matrix models in 2D quantum gravity. Comm. Math. Phys. 147 (1992), $395-430$.

[14] Ford, L. R., Automorphic Functions. New York: McGraw-Hill 1929.

[15] Fuchs, B. A. and Shabat, B. V., Functions of a Complex Variable and Some of Their Applications. Vol. 1. Oxford: Pergamon Press 1964.

[16] Gohberg, I., The factorization problem for operator functions (in Russian). Izv. Akad. Nauk SSSR, Ser. Mat. 28 (1964), 1055 - 1082.

[17] Gohberg, I., Kaashoek, M. A. and Spitkovskiı̌, I. M., An overview of matrix factorization theory and operator applications. In: Factorization and Integrable Systems (Faro 2000; ed.: I. Gohberg) Oper. Theory Adv. Appl. 141. Basel: Birkhäuser 2003, pp. 1 - 102.

[18] Ince, E. L., Ordinary Differential Equations. New York: Dover 1956.

[19] Khrapkov, A. A., Certain cases of the elastic equilibrium of an infinite wedge with a non-symmetric notch at the vertex, subject to concentrated force (in Russian). Prikl. Mat. Mech. 35 (1971), 677 - 689; transl. in: J. Appl. Mat. Mech. 35 (1971), 625 - 637. 
[20] Khvoshchinskaya, L. A., The homogeneous boundary value Riemann problem for two pairs of functions with the piecewise constant matrix in the case of four and more singular points (in Russian). Izv. Akad. Nauk Belarusi, Ser. Fiz.-Mat. Nauk 2 (1994), 124 - 125; (summary deposited at VINITI, No. 2400-B93).

[21] Kravchenko, V. G., Lebre, A. B. and Rodriguez, J. S., Factorization of singular integral operators with a Carleman shift and spectral problems. J. Integral Eqs. Appl. 13 (2001)(4), 339 - 383.

[22] Kythe, P. K., Computational Conformal Mapping. Boston: Birkhäuser 1998.

[23] Litvinchuk, G. S. and Spitkovskiǔ, I. M., Factorization of Measurable Matrix Functions. Oper. Theory: Adv. Appl. 25. Basel: Birkhäuser 1987.

[24] Meister, E. and Speck, F.-O., The explicit solution of elastodynamical diffraction problems by symbol factorization. Z. Anal. Anwendungen 8 (1989), 307 328.

[25] Mityushev, V. V. and Rogosin, S. V., Constructive Methods for Linear and Nonlinear Boundary Value Problems for Analytic Functions. Boca Raton (FL): Chapman \& Hall/ CRC 1999.

[26] Nehari, Z., Conformal Mappings. New York: McGraw-Hill 1952.

[27] Plemelj, J., Problems in the Sense of Riemann and Klein. New York: Interscience Publ. Wiley 1964.

[28] Schinzinger, R. and Laura, P. A. A., Conformal Mapping. Methods and Applications. Mineola (NY): Dover 2003.

[29] Vekua, N. P., Systems of Singular Integral Equations. Groningen: Noordhoff 1967.

[30] Zverovich, E. I., Boundary value problems of analytic functions in Hölder classes on Riemann surfaces (in Russian). Uspekhi Mat. Nauk 26 (1971) (1/157), 113 - 179; transl. in: Sovjet Math. Surv. 26 (1971), 117 - 192.

[31] Zverovich, E. I., An algebraic method for constructing the basic functional of a Riemann surface that is given in the form of a finite-sheeted covering of the sphere (in Russian). Sibirsk. Mat. Zh. 28 (1987)(6), 32 - 43; transl. in: Sib. Math. J. 28 (1987) (6) $889-898$.

[32] Zverovich, E. I. and Khvoshchinskaya, L. A., Solution of a generalized Riemann problem (in Russian). In: Proc. commemorative seminar on boundary value problems (eds.: V. P. Platonov et al.). Minsk: Univ. Publ. 1985, pp. 153 - 158. 